Article

\title{
Impact of Placement of Fitbit HR under Laboratory and Free-Living Conditions
}

\author{
Hyun Chul Jung ${ }^{1}\left(\mathbb{D}\right.$, Minsoo Kang ${ }^{2}{ }^{-}$, Nan Hee Lee ${ }^{3}$, Soeun Jeon ${ }^{3}$ and Sukho Lee ${ }^{3, *}$ \\ 1 Department of Coaching, College of Physical Education, Kyung Hee University-Global Campus, \\ 1732 Deogyeong-daero, Giheung-gu, Yongin-si, Gyeonggi-do 17014, Korea; jhc@khu.ac.kr \\ 2 Health and Sport Analytics Laboratory, Department of Health, Exercise Science, and Recreation Management, \\ The University of Mississippi, 219 Turner Center, University, MS 38677, USA; kang@olemiss.edu \\ 3 Department of Counseling, Health, and Kinesiology, College of Education and Human Development Texas \\ A\&M University-San Antonio, One University Way, San Antonio, TX 78224, USA; \\ nlee94@gatech.edu (N.H.L.); sonnyjeon@gmail.com (S.J.) \\ * Correspondence: slee@tamusa.edu; Tel.: +1-210-784-2537
}

Received: 30 June 2020; Accepted: 3 August 2020; Published: 5 August 2020

\begin{abstract}
Wrist-worn activity monitors have become accessible for measuring physical activities, but an activity monitor's accuracy worn at different placements is not well understood. This study aimed to examine the differences in measurements of heart rates, step counts, and calories estimated from the fitness tracker worn at different locations and the accuracy of Fitbit HR against criterion measures. Thirty-two healthy adults participated in this study. Participants wore Fitbit HR at four different locations (right proximal, distal, and left proximal, distal). Treadmill exercise consisted of five 5-min phases including slow walking and jogging. Free-living activities involved ten 5-min activities with four different intensities. We found that the placement of Fitbit HR has no significant influence on the measurement outcomes overall. However, significant differences were observed in the step counts between left and right while climbing stairs $(p=0.003)$ and sports activities $(p<0.001)$. The accuracy of Fitbit HR measurements against the criterion measure was moderate. However, we found out that Fitbit HR overestimated the calories regardless of their positions and activity types. In conclusion, the different placements of Fitbit HR do not have a significant influence on the measurements and Fitbit HR was moderately associated with criterion reference devices for measures of heart rate and steps.
\end{abstract}

Keywords: activity monitor; calories; heart rate; step; physical activity

\section{Introduction}

Physical activity (PA) has been recognized as an important factor for preventing cardiovascular diseases (CVDs) in the 21st century [1,2]. Because of PA's positive impact, various activity monitors that enable to self-monitor and track the PA level during free-living conditions have been introduced in the commercial market. The number of consumers increased year by year, and the wearable device market has grown from 84 million units sold in 2015 to 245 million units in 2019 [3]. Especially, wrist-worn activity monitors such as Fitbit HR, Garmin vívosmart, and smartwatches including Apple Watch and Samsung Gear have become popular due to their feasibility and multiple functions (i.e., phone call, text message, internet). For instance, Fitbit (Fitbit Inc., San Francisco, CA, USA) is one of the leading brands among activity monitors that have reached more than 27.4 million active application users in the United States in 2018 [4].

Fitbit Flex, the first wrist-worn activity monitor, produced by the Fitbit company, was introduced in 2013 and provided general activity information such as step counts and sleep duration. As the devices 
have evolved by advanced technologies, they nowadays provide PA feedback information of various forms including step counts, calories, heart rate, and traveled distance. Particularly, heart rate is a specific feature of the wrist-worn activity monitor. The monitor utilizes an optical sensor that estimates heart rate via photoplethysmography (PPG) technique and this non-invasive technique probes small variations of pulsatile blood flow under the skin [5]. Heart rate estimation allows monitoring exercise intensity in real time and provides more accurate exercise intensity than accelerometers at non-weight bearing exercise setting (i.e., cycling and swimming) [6]. However, heart rate has an inherent feature as a vital sign, fluctuated by mood, emotional status, and illness that may limit to monitor the accurate exercise intensity or recovery.

It has been demonstrated that the application of activity monitors increases consumers' PA level and modifies their health and sleep behaviors [7,8]. Still, there is a significant challenge in the research and clinical environments, regarding whether the obtained data under free-living conditions are valid and reliable. A number of validity studies have been conducted to examine the accuracy of step counts and calories [9-12]. Step count is one of the fundamental functions of activity monitors. Many studies have shown that wrist-worn activity monitors accurately assessed steps in the laboratory and free-living settings [11-14]. However, a recent study found that the mean absolute percent errors against researcher-counted steps were higher in some activities such as cycling and climbing stairs than walking or jogging [15]. Especially, conflicting information has been observed regarding heart rate sensors. While a moderate to strong correlation against the criterion measure was observed under the laboratory conditions [5], the accuracy decreased during moderate to vigorous activities in free-living conditions [6]. Another underlying issue is the location of the device. While the versatility of altering the wearing locations (i.e., left vs. right; distal vs. proximal) may prove appealing, monitor placement on the outcome measurements needs to be examined. Therefore, the present study aimed to examine (1) the difference in measurements of heart rate, step counts, and calories estimated from the Fitbit HR worn at different locations, and (2) the accuracy of Fitbit HR against criterion measures during lab and free-living activities.

\section{Materials and Methods}

\subsection{Experimental Design}

First, the health status of all participants was screened with the Physical Activity Readiness Questionnaire (PAR-Q) and the participants' physique, blood pressure, and three-site skinfold thickness were measured. The participants then wore Fitbit HR at four different locations to the left and right wrists (right proximal (RP), right distal (RD), left proximal (LP), left distal (LD)). Each participant performed physical activities in two different conditions including the lab and free-living settings.

\subsection{Participants}

Initially, thirty-five healthy males and females, aged 18-40 years, enrolled in this study. The inclusion criteria for participants were as follows: (1) voluntary participation, (2) ability to participate in physical activities, and (3) not having had any skeletomuscular injuries for the last three months. Three participants who did not complete the whole study procedure were excluded from the study; therefore, data form thirty-two participants were analyzed in this study. The sample size was calculated based on a $95 \%$ confidence interval, a medium effect size $(\mathrm{f}=0.25)$, and a power of $90 \%$ to detect the difference among the various locations. The power analysis results showed that at least 30 participants were needed in the experiment. Each participant completed the written consent form that was approved by the Institutional Review Board of the University.

\subsection{Measurements}

Physique, blood pressure, and heart rate: Participants' height and body weight were measured with a stadiometer (PAT \#290237, Novel Products, St. Paul, MN, USA) and a digital scale (HD-366, 
Tanita, Tokyo, Japan). The body mass index was calculated as kilogram per square meter. Three skinfold thickness sites were measured by caliper (Beta Technology, Santa Cruz, CA, USA). The skinfold sites in male participants were chest, abdominal, and thigh while for female participants they were triceps, suprailiac, and thigh. Each participant was measured three times on the right side of the participant's body, and the average score was recorded. The results were then converted into body density and body fat percentages by Jackson and Pollock and Siri's equations [16,17]. Resting heart rate and blood pressure was measured by an autonomic sphygmomanometer (BP791IT, Omron, Kyoto, Japan).

Treadmill exercise test: The treadmill exercise test was modified from a previous study [18]. The participant wore Fitbit HR at four different locations and sat quietly for 5 minutes on a chair. The test consisted of five 5-minute phases including a resting phrase, slow walking $(53.6 \mathrm{~m} / \mathrm{min})$, brisk walking $(107.3 \mathrm{~m} / \mathrm{min})$, jogging $(160.9 \mathrm{~m} / \mathrm{min})$, and a recovery phrase. Finally, they sat on the chair for 5 minutes as a recovery phase. Heart rate, calories, and step counts for each phase were recorded from four Fitbit HR, while metabolic cart (TrueOne 2400, Medics, Salt Lake City, UT, USA) was used for calories, a chest-strap heart rate monitor (Polar, Bethpage, NY, USA) for heart rate, and a digital camera (Digital camera, Kodak, Rochester, NY, USA) with a hand tally counter for step counts were used as criterion measures to compare with Fitbit HR.

Free-living activities: Participants performed ten 5-min simulated physical activities similarly designed to free-living conditions. The activity protocols were modified from previous studies $[19,20]$. The activities were divided into four categories including sedentary, light ( $<3 \mathrm{MET})$, moderate (3-6MET), and vigorous activities ( $>6 \mathrm{MET}$ ) based on metabolic equivalent (MET) intensity. Each participant performed the activities for 50 minutes. The average heart rate, calories, and steps in each phase were recorded from Fitbit HR. ActiGraph GT3X (ActiGraph LLC, Pensacola, FL, USA) was used for estimating calories as a criterion measure in the free-living activities. The accuracy of the ActiGraph GT3X was validated in the previous study [21]. The participant wore the ActiGraph GT3X on the right side of the waist and collected the data during free-living activities. The heart rate and steps were measured in the same manner applied for the treadmill exercise test. Simulated free-living activities are shown in Table 1.

Table 1. Simulated Free-Living Activities.

\begin{tabular}{cccc}
\hline Activity & Intensity & Duration & Descriptive Activity \\
\hline Lying down & Sedentary & $5 \mathrm{~min}$ & Lying on a bed \\
Shopping & Light & $5 \mathrm{~min}$ & Pushing a cart \\
Climbing Stairs & Moderate & $5 \mathrm{~min}$ & Climbing using step boxes \\
Laundry & Light & $5 \mathrm{~min}$ & Folding towels \\
Cleaning & Moderate & $5 \mathrm{~min}$ & Mopping the laboratory \\
Computer & Sedentary & $5 \mathrm{~min}$ & Surfing the web \\
Stretching & Light & $5 \mathrm{~min}$ & Stretching \\
Walking & Light & $5 \mathrm{~min}$ & Walking around the school \\
Jogging & Vigorous & $5 \mathrm{~min}$ & Jogging around school \\
Sports activity & Vigorous & $5 \mathrm{~min}$ & Basketball dribbling \\
\hline
\end{tabular}

Activity monitor: Fitbit Charge HR (Fitbit Inc., San Francisco, CA, USA) was used in the study. Four Fitbit HR were placed on the right and left wrists. The first device was placed on the $2 \mathrm{~cm}$ above participant's left wrist bone. The second device was placed $2 \mathrm{~cm}$ above from the first device. The third and fourth devices were placed in the same manner on the right wrist. The location of the devices was systematically rotated. Before experimenting, participants' individual information including height, body weight, and birthday were set up via the Fitbit Android application.

\subsection{Data Analysis}

Descriptive statistics were computed for all variables and presented as mean and standard deviation for continuous variables and frequency and percentage for categorical variables, respectively. 
To compare measurements among various wearing positions of Fitbit HR, repeated measures ANOVAs were performed across four different wearing positions (i.e., RP, RD, LP, and LD) under laboratory and free-living settings for each outcome measurement: heart rate, step counts, and calorie. The significance of the repeated measures ANOVAs was examined using Bonferroni adjusted alpha levels, which was 0.003 (i.e., 0.05/15). To examine the accuracy of Fitbit HR worn at different positions against criterion measure, the mean difference (MD) was calculated by subtracting criterion-measured values of heart rate, step counts, and calorie from the estimated values of each Fibit HR for various activities and settings. Negative MD indicates underestimation compared to a criterion measure, while positive MD indicates overestimation. The $95 \%$ confidence interval (CI) of MD was calculated to determine the statistical significance of MD. If 95\% CI did not include $0, \mathrm{MD}$ was considered a significant difference. For additional information, the MD's effect size was evaluated using Cohen's $d$ (i.e., MD/standard deviation of MD). All statistical analyses were performed using SPSS Version 23 (SPSS Inc, Chicago, IL, USA).

\section{Results}

Data collected from this study included 7200 data points (225 variables * 32 participants). During data screening, few participants with invalid Fitbit monitor reading values (i.e., negative values in heart rate, step counts, and calorie) were identified. The anomalous values were 24 data points, which is about $0.3 \%$ of the total data points. The invalid values were set to missing to preserve the remaining data. As a result, samples of 29 to 32 were used across all data analyses in this study. Of all participants $(n=32), 46.88 \%$ were males, and $96.88 \%$ were right-handed. The average age and body mass index were $26.03 \pm 6.59$ years and $26.40 \pm 5.11 \mathrm{~kg} / \mathrm{m} 2$, respectively. The detailed descriptive statistics of the participants are presented in Table 2.

Table 2. Characteristics of Participants $(n=32)$.

\begin{tabular}{ccc}
\hline Variables & Frequency & Percent \\
\hline Men & 15.00 & 46.88 \\
Women & 17.00 & 53.13 \\
Right-handed & 31.00 & 96.88 \\
Left-handed & 1.00 & 3.13 \\
& Mean & SD \\
Age (years) & 26.03 & 6.59 \\
Height $(\mathrm{cm})$ & 167.38 & 8.30 \\
Weight $(\mathrm{kg})$ & 74.09 & 15.89 \\
Body mass index $\left(\mathrm{kg} / \mathrm{m}^{2}\right)$ & 26.40 & 5.11 \\
Body fat percentage $(\%)$ & 21.09 & 7.73 \\
Systolic blood pressure $(\mathrm{mmHg})$ & 119.28 & 14.59 \\
Diastolic blood pressure $(\mathrm{mmHg})$ & 75.00 & 7.48 \\
HR (beats/minute) & 73.41 & 10.17 \\
\hline
\end{tabular}

Overall, there were no differences in measurements of heart rate, step counts, and calories estimated from the four Fitbit HR worn at different positions (Table 3). In a free-living setting, step counts were significantly different for climbing stairs $(p=0.003)$ and sports activities $(p<0.001)$. Step counts estimated from Fitbit HR worn at the distal position of the left wrist were significantly higher than from Fitbit HR worn at the proximal and distal positions of the right wrist when climbing stairs. Fitbit HR worn on the left wrist estimated significantly higher step counts than Fitbit HR worn on the right wrist while participating in sports (Table 3).

The accuracy of Fitbit HR was moderate in heart rate estimation, especially during low-intensity activities (Table 4). Fitbit HR tended to underestimate heart rate compared to a criterion measure, particularly during moderate- to vigorous-intensity activities including walking, jogging, and sports. Heart rate estimated from Fitbit HR worn at the proximal position of the wrist was slightly more 
accurate than heart rate estimated from Fitbit HR worn at the distal position of the wrist in a laboratory setting, but there was no similar pattern or tendency identified in the free-living condition.

Moderate accuracy was found in measurements of step counts in the laboratory setting, but poor accuracy was observed in the free-living setting (Table 5). In the laboratory setting, all Fitbit HR showed good accuracy in estimating step counts during moderate- to vigorous-intensity activities, such as brisk walking and jogging. On the other hand, poor accuracy was identified across all Fitbit HR during shopping and laundry in a free-living setting. No noticeable pattern or tendency was identified in the laboratory and free-living settings for step counts.

Overall, the accuracy of Fitbit HR was poor for calorie estimation (Table 6). Fitbit HR tended to overestimate calories when compared to criterion measures regardless of their positions and activity types. 
Table 3. Comparisons of Heart Rate, Steps, and Calories Estimated from Four Fitbits in Laboratory and Free-Living Settings.

\begin{tabular}{|c|c|c|c|c|c|c|c|c|}
\hline & \multirow{2}{*}{ Settings and Activities } & \multirow{2}{*}{ Variables } & \multirow{2}{*}{$\mathrm{n}$} & \multicolumn{2}{|c|}{ Right Wrist } & \multicolumn{2}{|c|}{ Left Wrist } & \multirow{2}{*}{$p$-Values } \\
\hline & & & & Proximal (RP) & Distal (RD) & Proximal (LP) & Distal (LD) & \\
\hline \multirow{15}{*}{ Lab Activity } & \multirow{3}{*}{ Resting } & Heart Rate & 32 & $76.84(11.53)$ & $76.00(11.16)$ & $77.69(11.82)$ & $76.34(10.61)$ & 0.159 \\
\hline & & Steps & 32 & $0.22(1.24)$ & $0.13(0.71)$ & $0.00(0.00)$ & $0.00(0.00)$ & 0.325 \\
\hline & & Calories & 32 & $4.94(3.45)$ & $15.03(60.97)$ & $4.31(1.53)$ & $4.56(2.06)$ & 0.341 \\
\hline & \multirow{3}{*}{ Slow walking ( $2 \mathrm{mph})$} & Heart rate & 32 & $100.56(14.48)$ & $98.88(12.06)$ & $99.50(16.50)$ & $96.72(12.08)$ & 0.594 \\
\hline & & Steps & 32 & $279.94(32.25)$ & $281.19(34.21)$ & $272.78(45.17)$ & $280.78(30.47)$ & 0.370 \\
\hline & & Calories & 31 & $20.61(5.49)$ & $22.03(6.47)$ & $21.81(6.52)$ & $21.39(6.35)$ & 0.475 \\
\hline & \multirow{4}{*}{ Brisk Walking (4 mph) } & Heart Rate & 32 & $130.03(20.61)$ & $123.34(16.07)$ & $126.19(20.04)$ & $122.06(16.46)$ & 0.076 \\
\hline & & Steps & 32 & 406.75 (80.69) & $380.84(69.53)$ & $399.50(95.40)$ & $384.19(64.33)$ & 0.140 \\
\hline & & Calories & 32 & $28.66(7.26)$ & $29.63(10.18)$ & $29.47(9.52)$ & $31.22(12.06)$ & 0.516 \\
\hline & & Heart rate & 31 & $160.65(16.35)$ & $153.61(18.31)$ & $161.84(16.26)$ & $157.29(19.55)$ & 0.045 \\
\hline & \multirow[t]{2}{*}{ Jogging (6 mph) } & Steps & 32 & $477.03(24.73)$ & $472.97(26.80)$ & $478.25(25.49)$ & $478.81(25.71)$ & 0.033 \\
\hline & & Calories & 30 & 40.73 (13.49) & 41.87 (19.56) & 40.47 (17.53) & 42.83 (17.07) & 0.765 \\
\hline & \multirow{3}{*}{ Recovery } & Heart rate & 32 & $96.81(20.99)$ & $93.63(15.89)$ & 94.41 (15.88) & $94.19(15.81)$ & 0.170 \\
\hline & & Steps & 31 & $3.68(5.50)$ & $3.10(5.87)$ & $3.10(5.20)$ & $3.55(6.11)$ & 0.732 \\
\hline & & Calories & 31 & 27.19 (13.69) & $37.03(56.67)$ & $28.26(21.68)$ & $25.23(13.81)$ & 0.342 \\
\hline \multirow{30}{*}{ Free-living setting } & \multirow{3}{*}{ Lying down } & Heart rate & 32 & $78.72(10.90)$ & $78.44(11.28)$ & $79.34(11.58)$ & $78.69(10.86)$ & 0.369 \\
\hline & & Steps & 32 & $0.28(1.11)$ & $0.28(1.11)$ & $0.91(4.45)$ & $0.75(3.73)$ & 0.482 \\
\hline & & Calories & 30 & $7.82(3.89)$ & $8.28(4.11)$ & $8.25(5.36)$ & $8.17(5.08)$ & 0.686 \\
\hline & \multirow{3}{*}{ Shopping } & Heart rate & 32 & $90.97(11.38)$ & $89.25(8.86)$ & 87.97 (14.73) & 88.94 (13.12) & 0.287 \\
\hline & & Steps & 32 & 55.16 (91.48) & 64.69 (93.64) & 40.66 (70.71) & 58.09 (91.87) & 0.457 \\
\hline & & Calories & 29 & $13.93(8.49)$ & $14.93(7.19)$ & 16.69 (24.04) & $14.07(8.36)$ & 0.646 \\
\hline & \multirow{3}{*}{ Climbing stairs } & Heart rate & 32 & 133.19 (21.77) & $132.25(20.96)$ & $126.78(21.80)$ & $128.94(22.56)$ & 0.128 \\
\hline & & Steps & 32 & $413.47(60.84)$ & $417.47(49.26)$ & $438.34(50.80)$ & $445.97(48.65)$ & 0.003 * \\
\hline & & Calories & 32 & $36.28(12.04)$ & $45.47(39.82)$ & $36.31(9.06)$ & $53.22(69.65)$ & 0.260 \\
\hline & \multirow{3}{*}{ Laundry } & Heart rate & 32 & $100.66(16.32)$ & $102.63(12.54)$ & $100.03(14.76)$ & $101.19(16.45)$ & 0.474 \\
\hline & & Steps & 31 & $184.00(68.74)$ & 178.77 (78.27) & 181.10 (88.79) & 177.13 (91.17) & 0.867 \\
\hline & & Calories & 30 & $34.53(12.88)$ & 35.80 (15.24) & 33.57 (14.67) & 34.40 (16.96) & 0.561 \\
\hline & \multirow{3}{*}{ Cleaning } & Heart rate & 32 & 98.00 (16.98) & 98.94 (16.09) & 96.72 (17.48) & 97.38 (16.86) & 0.603 \\
\hline & & Steps & 32 & $232.72(364.24)$ & $184.47(126.93)$ & $171.81(116.00)$ & 190.47 (125.05) & 0.429 \\
\hline & & Calories & 30 & $22.93(11.48)$ & 24.13 (13.63) & 23.63 (11.31) & 24.87 (13.26) & 0.565 \\
\hline & \multirow{4}{*}{ Computer } & Heart rate & 32 & 78.22 (11.73) & 78.41 (12.77) & $79.53(12.60)$ & 79.59 (12.49) & 0.069 \\
\hline & & Steps & 32 & $1.53(3.84)$ & $2.63(5.93)$ & $1.47(4.11)$ & $1.16(3.55)$ & 0.393 \\
\hline & & Calories & 31 & $17.42(8.85)$ & 18.55 (11.74) & $18.71(12.06)$ & 19.48 (14.07) & 0.200 \\
\hline & & Heart rate & 32 & $97.06(15.76)$ & $98.28(14.60)$ & $97.28(16.03)$ & 96.84 (16.19) & 0.834 \\
\hline & \multirow[t]{2}{*}{ Stretching } & Steps & 32 & 30.00 (19.41) & 47.25 (101.11) & 39.09 (17.27) & 37.25 (16.46) & 0.460 \\
\hline & & Calories & 31 & $15.87(9.13)$ & 17.58 (10.65) & $16.97(8.80)$ & 18.13 (10.49) & 0.295 \\
\hline & \multirow{4}{*}{ Walking } & Heart rate & 32 & $101.13(14.21)$ & $100.78(14.96)$ & $101.16(14.28)$ & $101.72(16.32)$ & 0.882 \\
\hline & & Steps & 31 & 468.48 (51.92) & $465.16(55.31)$ & 473.35 (36.61) & 470.68 (39.45) & 0.453 \\
\hline & & Calories & 32 & $33.38(9.36)$ & 36.78 (11.70) & $35.78(10.60)$ & 37.34 (12.54) & 0.058 \\
\hline & & Heart rate & 31 & 151.19 (19.89) & 150.00 (20.34) & 153.48 (20.57) & 151.35 (21.70) & 0.598 \\
\hline & Jogging & Steps & 32 & 778.81 (119.14) & 751.22 (42.06) & 760.47 (54.90) & $757.16(62.36)$ & 0.258 \\
\hline & & Calories & 32 & 74.69 (129.39) & $51.88(18.96)$ & $63.03(38.76)$ & $59.28(25.08)$ & 0.451 \\
\hline & & Heart rate & 30 & 151.47 (19.50) & 149.07 (19.33) & $156.83(21.73)$ & $154.20(22.46)$ & 0.113 \\
\hline & Sports & Steps & 32 & 586.75 (75.02) & 565.34 (72.73) & 648.84 (64.43) & 641.66 (69.86) & $<0.001^{*}$ \\
\hline & & Calories & 29 & $55.76(19.27)$ & $60.24(20.30)$ & $59.93(21.14)$ & $62.66(22.68)$ & 0.260 \\
\hline
\end{tabular}

Note: Data are presented as mean (standard deviation); LD = left distal; LP = left proximal; $\mathrm{RD}=$ right distal; $\mathrm{RP}=$ right proximal; ${ }^{*}$ indicates significant difference among means $(p<0.004)$. 
Table 4. Comparisons of Heart Rate Estimated by Four Fitbits with Criterion-Measured Heart Rate in the Laboratory and Free-Living Settings.

\begin{tabular}{|c|c|c|c|c|c|c|c|}
\hline & \multirow{2}{*}{ Settings and Activities } & \multirow{2}{*}{ Variables } & \multicolumn{2}{|c|}{ Right Wrist } & \multicolumn{2}{|c|}{ Left Wrist } & \multirow{2}{*}{ Criterion Measure } \\
\hline & & & Proximal (RP) & Distal (RD) & Proximal (LP) & Distal (LD) & \\
\hline \multirow{5}{*}{ Lab Activity } & Resting & $\begin{array}{l}\text { MD }(95 \% \text { CI }) \\
\text { Effect Size }\end{array}$ & $\begin{array}{c}\mathbf{- 1 . 7 5}(-3.42,-0.08) \\
-0.38\end{array}$ & $\begin{array}{c}-2.59(-4.35,-0.84) \\
-0.53\end{array}$ & $\begin{array}{c}-0.91(-2.68,0.87) \\
-0.18\end{array}$ & $\begin{array}{c}-2.25(-4.13,-0.37) \\
-0.43\end{array}$ & 78.59 (12.41) \\
\hline & Slow walking (2 mph) & $\begin{array}{l}\text { MD ( } 95 \% \text { CI) } \\
\text { Effect Size }\end{array}$ & $\begin{array}{c}2.72(-2.94,8.37) \\
0.17\end{array}$ & $\begin{array}{c}1.03(-4.21,6.27) \\
0.07\end{array}$ & $\begin{array}{c}1.66(-3.72,7.04) \\
0.11\end{array}$ & $\begin{array}{c}-1.13(-4.92,2.67) \\
-0.11\end{array}$ & $97.84(12.42)$ \\
\hline & Brisk walking (4 mph) & $\begin{array}{l}\text { MD ( } 95 \% \text { CI }) \\
\text { Effect Size }\end{array}$ & $\begin{array}{c}0.28(-5.78,6.34) \\
0.02\end{array}$ & $\begin{array}{c}-6.41(-9.50,-3.31) \\
-0.75\end{array}$ & $\begin{array}{c}-3.56(-9.87,2.74) \\
-0.20\end{array}$ & $\begin{array}{c}-7.69(-11.56,-3.81) \\
-0.72\end{array}$ & $129.75(17.51)$ \\
\hline & Jogging (6 mph) & $\begin{array}{l}\text { MD }(95 \% \text { CI }) \\
\text { Effect Size }\end{array}$ & $\begin{array}{c}\mathbf{- 9 . 0 0}(-\mathbf{1 2 . 3 7},-5.63) \\
-0.96\end{array}$ & $\begin{array}{c}-15.84(-22.39,-9.30) \\
-0.87\end{array}$ & $\begin{array}{c}-8.63(-12.51,-4.74) \\
-0.80\end{array}$ & $\begin{array}{c}-12.61(-18.82,-6.40) \\
-0.74\end{array}$ & $169.59(17.31)$ \\
\hline & Recovery & $\begin{array}{l}\text { MD }(95 \% \text { CI }) \\
\text { Effect Size }\end{array}$ & $\begin{array}{c}0.28(-4.35,4.91) \\
0.02\end{array}$ & $\begin{array}{c}-2.91(-5.32,-0.49) \\
-0.43\end{array}$ & $\begin{array}{c}-2.13(-4.50,0.25) \\
-0.32\end{array}$ & $\begin{array}{c}-2.34(-4.51,-0.17) \\
-0.39\end{array}$ & $96.53(17.19)$ \\
\hline \multirow{10}{*}{ Free-living Setting } & Lying down & $\begin{array}{l}\text { MD (95\% CI) } \\
\text { Effect Size }\end{array}$ & $\begin{array}{c}-0.78(-2.29,0.73) \\
-0.19\end{array}$ & $\begin{array}{c}-1.06(-2.58,0.45) \\
-0.25\end{array}$ & $\begin{array}{c}-0.16(-1.38,1.07) \\
-0.05\end{array}$ & $\begin{array}{c}-0.81(-2.43,0.80) \\
-0.18\end{array}$ & $79.5(12.35)$ \\
\hline & Shopping & $\begin{array}{l}\text { MD }(95 \% \text { CI }) \\
\text { Effect Size }\end{array}$ & $\begin{array}{c}-4.66(-7.29,-2.02) \\
-0.64\end{array}$ & $\begin{array}{c}-6.38(-9.87,-2.88) \\
-0.66\end{array}$ & $\begin{array}{c}-7.66(-11.08,-4.23) \\
-0.81\end{array}$ & $\begin{array}{c}-6.69(-9.23,-4.14) \\
-0.95\end{array}$ & $95.63(13.93)$ \\
\hline & Climbing stairs & $\begin{array}{l}\text { MD }(95 \% \text { CI }) \\
\text { Effect Size }\end{array}$ & $\begin{array}{c}-0.75(-6.38,4.88) \\
-0.05\end{array}$ & $\begin{array}{c}-1.69(-6.13,2.76) \\
-0.14\end{array}$ & $\begin{array}{c}-7.16(-15.06,0.75) \\
-0.33\end{array}$ & $\begin{array}{c}-5.00(-10.86,0.86) \\
-0.31\end{array}$ & $133.94(24.63)$ \\
\hline & Laundry & $\begin{array}{l}\text { MD }(95 \% \text { CI }) \\
\text { Effect Size }\end{array}$ & $\begin{array}{c}-2.53(-5.99,0.93) \\
-0.26\end{array}$ & $\begin{array}{c}-0.56(-4.66,3.53) \\
-0.05\end{array}$ & $\begin{array}{c}-3.16(-7.60,1.29) \\
-0.26\end{array}$ & $\begin{array}{c}-2.00(-5.71,1.71) \\
-0.19\end{array}$ & $103.19(18.39)$ \\
\hline & Cleaning & $\begin{array}{l}\text { MD }(95 \% \text { CI }) \\
\text { Effect Size }\end{array}$ & $\begin{array}{c}-2.84(-6.47,0.78) \\
-0.28\end{array}$ & $\begin{array}{c}-1.91(-5.57,1.76) \\
-0.19\end{array}$ & $\begin{array}{c}-4.13(-8.00,-0.25) \\
-0.38\end{array}$ & $\begin{array}{c}-3.47(-7.79,0.85) \\
-0.29\end{array}$ & $100.84(17.84)$ \\
\hline & Computer & $\begin{array}{l}\text { MD }(95 \% \text { CI }) \\
\text { Effect Size }\end{array}$ & $\begin{array}{c}-1.59(-3.55,0.37) \\
-0.29\end{array}$ & $\begin{array}{c}-1.41(-3.23,0.41) \\
-0.28\end{array}$ & $\begin{array}{c}-0.28(-2.04,1.47) \\
-0.06\end{array}$ & $\begin{array}{c}-0.22(-2.07,1.64) \\
-0.04\end{array}$ & $79.81(12.09)$ \\
\hline & Stretching & $\begin{array}{l}\text { MD (95\% CI) } \\
\text { Effect Size }\end{array}$ & $\begin{array}{c}-1.31(-5.07,2.45) \\
-0.13\end{array}$ & $\begin{array}{c}-0.09(-4.05,3.86) \\
\quad-0.01\end{array}$ & $\begin{array}{c}-1.09(-4.30,2.12) \\
-0.12\end{array}$ & $\begin{array}{c}-1.53(-4.67,1.61) \\
-0.18\end{array}$ & $98.38(17.98)$ \\
\hline & Walking & $\begin{array}{l}\text { MD }(95 \% \text { CI }) \\
\text { Effect Size }\end{array}$ & $\begin{array}{c}-2.66(-5.14,-0.17) \\
-0.39\end{array}$ & $\begin{array}{c}-3.00(-5.36,-0.64) \\
-0.46\end{array}$ & $\begin{array}{c}-2.63(-6.32,1.07) \\
-0.26\end{array}$ & $\begin{array}{c}-2.06(-5.58,1.46) \\
-0.21\end{array}$ & $103.78(16.99)$ \\
\hline & Jogging & $\begin{array}{l}\text { MD }(95 \% \text { CI }) \\
\text { Effect Size }\end{array}$ & $\begin{array}{c}-8.16(-15.35,-0.96) \\
-0.41\end{array}$ & $\begin{array}{c}\mathbf{- 9 . 1 6}(-\mathbf{1 4 . 4 3 ,}-3.88) \\
-0.63\end{array}$ & $\begin{array}{c}-5.78(-10.16,-1.41) \\
-0.48\end{array}$ & $\begin{array}{c}-7.13(-12.52,-1.73) \\
-0.48\end{array}$ & $159.09(22.3)$ \\
\hline & Sports & $\begin{array}{l}\text { MD }(95 \% \text { CI }) \\
\text { Effect Size }\end{array}$ & $\begin{array}{c}-13.91(-20.39,-7.42) \\
-0.77\end{array}$ & $\begin{array}{c}\mathbf{- 1 7 . 0 0}(\mathbf{- 2 3 . 5 0 , - 1 0 . 5 )} \\
-0.94\end{array}$ & $\begin{array}{c}-10.87(-16.04,-5.70) \\
-0.77\end{array}$ & $\begin{array}{c}-11.39(-16.14,-6.64) \\
-0.88\end{array}$ & $166.03(18.62)$ \\
\hline
\end{tabular}

Note: Data are presented as mean difference (95\% confidence interval); $\mathrm{MD}=$ mean difference; $\mathrm{MD}$ is calculated by subtracting the criterion measured value from each Fitbit measured value; $\mathrm{LD}=$ left distal; $\mathrm{LP}=$ left proximal; $\mathrm{RD}=$ right distal; $\mathrm{RP}=$ right proximal; Sample sizes $=31-32$; The bold numbers indicate significant difference from zero $(p<0.05)$. 
Table 5. Comparisons of Steps Estimated by Four Fitbits with Criterion-Measured Steps in the Laboratory and Free-Living Settings.

\begin{tabular}{|c|c|c|c|c|c|c|c|}
\hline & \multirow{2}{*}{ Settings and Activities } & \multirow{2}{*}{ Variables } & \multicolumn{2}{|c|}{ Right Wrist } & \multicolumn{2}{|c|}{ Left Wrist } & \multirow{2}{*}{ Criterion Measure } \\
\hline & & & Proximal (RP) & Distal (RD) & Proximal (LP) & Distal (LD) & \\
\hline \multirow{5}{*}{ Lab Activity } & Resting & $\begin{array}{l}\mathrm{MD}(95 \% \mathrm{CI}) \\
\text { Fffect Size }\end{array}$ & $0.22(-0.23,0.66)$ & $0.13(-0.13,0.38)$ & $0(0,0)$ & $0(0,0)$ & $0.00(0.00)$ \\
\hline & Slow walking (2 mph) & $\begin{array}{l}\text { MD }(95 \% \text { CI }) \\
\text { Effect Size }\end{array}$ & $\begin{array}{c}-13.47(-25.39,-1.55) \\
-0.41\end{array}$ & $\begin{array}{c}0.18 \\
-12.22(-25.21,0.78) \\
-0.34\end{array}$ & $\begin{array}{c}\mathrm{N} / \mathrm{A} \\
-20.63(-37.80,-3.45) \\
-0.43\end{array}$ & $\begin{array}{c}\mathrm{N} / \mathrm{A} \\
-12.63(-23.70,-1.55) \\
-0.41\end{array}$ & $293.41(25.95)$ \\
\hline & Brisk walking(4 mph) & $\begin{array}{l}\text { MD (95\% CI) } \\
\text { Effect Size }\end{array}$ & $\begin{array}{c}19.84(-9.05,48.74) \\
0.25\end{array}$ & $\begin{array}{c}-6.06(-31.48,19.36) \\
-0.09\end{array}$ & $\begin{array}{c}12.59(-21.49,46.68) \\
0.13\end{array}$ & $\begin{array}{c}-2.72(-25.67,20.24) \\
-0.04\end{array}$ & $386.91(18.39)$ \\
\hline & Jogging(6 mph) & $\begin{array}{l}\text { MD }(95 \% \text { CI }) \\
\text { Effect Size }\end{array}$ & $\begin{array}{c}-6.88(-18.36,4.61) \\
-0.22\end{array}$ & $\begin{array}{c}-10.94(-24.13,2.26) \\
-0.30\end{array}$ & $\begin{array}{c}-5.66(-17.27,5.95) \\
-0.18\end{array}$ & $\begin{array}{c}-5.09(-16.33,6.14) \\
-0.16\end{array}$ & $483.91(35.94)$ \\
\hline & Recovery & $\begin{array}{c}\text { MD }(95 \% \text { CI }) \\
\text { Effect Size }\end{array}$ & $\begin{array}{c}3.5(1.51,5.49) \\
0.64\end{array}$ & $\begin{array}{c}2.94(0.83,5.05) \\
0.50\end{array}$ & $\begin{array}{c}3.63(1.40,5.85) \\
0.59\end{array}$ & $\begin{array}{c}3.48(1.23,5.74) \\
0.56\end{array}$ & $0.06(0.35)$ \\
\hline \multirow{11}{*}{ Free-living Setting } & Lying down & $\begin{array}{l}\text { MD (95\% CI) } \\
\text { Effect Size }\end{array}$ & $\begin{array}{c}0.53(-0.22,1.29) \\
0.25\end{array}$ & $\begin{array}{c}0.53(-0.22,1.29) \\
0.25\end{array}$ & $\begin{array}{c}1.81(-1.40,5.02) \\
0.20\end{array}$ & $\begin{array}{c}1.47(-1.16,4.10) \\
0.20\end{array}$ & $0.00(0.00)$ \\
\hline & Shopping & $\begin{array}{c}\text { MD }(95 \% \text { CI }) \\
\text { Effect Size }\end{array}$ & $\begin{array}{c}-303.56(-342.20 \\
-264.93) \\
-2.83\end{array}$ & $\begin{array}{c}-294.03(-335.93 \\
-252.13) \\
-2.53\end{array}$ & $\begin{array}{c}-318.06(-354.49 \\
-281.64) \\
-3.15\end{array}$ & $\begin{array}{c}-300.63(-344.35 \\
-256.90) \\
-2.48\end{array}$ & $358.72(90.24)$ \\
\hline & Climbing stairs & $\begin{array}{l}\text { MD }(95 \% \text { CI }) \\
\text { Effect Size }\end{array}$ & $\begin{array}{c}-16.41(-38.53,5.72) \\
-0.27\end{array}$ & $\begin{array}{c}-12.41(-31.15,6.33) \\
-0.24\end{array}$ & $\begin{array}{c}8.47(-10.39,27.32) \\
0.16\end{array}$ & $\begin{array}{c}16.09(-1.72,33.91) \\
0.33\end{array}$ & $429.88(11.18)$ \\
\hline & Laundry & $\begin{array}{l}\text { MD }(95 \% \text { CI }) \\
\text { Effect Size }\end{array}$ & $\begin{array}{c}154.03(130.15,177.91) \\
2.37\end{array}$ & 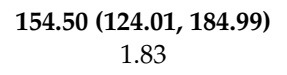 & $\begin{array}{c}154.09(\mathbf{1 2 7 . 9 4 ,} 180.25) \\
2.12\end{array}$ & $\begin{array}{c}149.41(\mathbf{1 1 9 . 9 0}, \mathbf{1 7 8 . 9 2}) \\
1.83\end{array}$ & $30.41(33.29)$ \\
\hline & Cleaning & $\mathrm{MD}(95 \% \mathrm{CI})$ & $-6.19(-143.93,131.55)$ & $-54.44(-109.27,0.39)$ & $\begin{array}{c}-67.09(-110.68 \\
-23.51)\end{array}$ & $-48.44(-96.23,-0.65)$ & $238.91(65.28)$ \\
\hline & & Effect Size & -0.02 & -0.36 & -0.56 & -0.37 & \\
\hline & Computer & $\begin{array}{l}\text { MD }(95 \% \text { CI }) \\
\text { Effect Size }\end{array}$ & $\begin{array}{c}1.53(\mathbf{0 . 1 5 , 2 . 9 2 )} \\
0.40\end{array}$ & $\begin{array}{c}2.63(0.49,4.76) \\
0.44\end{array}$ & $\begin{array}{c}1.47(-0.01,2.95) \\
0.36\end{array}$ & $\begin{array}{c}1.16(-0.12,2.44) \\
0.33\end{array}$ & $0.00(0.00)$ \\
\hline & Stretching & $\begin{array}{l}\text { MD }(95 \% \text { CI }) \\
\text { Effect Size }\end{array}$ & $\begin{array}{c}-10.97(-19.56,-2.38) \\
-0.46\end{array}$ & $\begin{array}{c}6.28(-30.95,43.51) \\
0.06\end{array}$ & $\begin{array}{c}-1.88(-9.11,5.36) \\
-0.09\end{array}$ & $\begin{array}{c}-3.72(-10.35,2.91) \\
-0.20\end{array}$ & $40.97(11.62)$ \\
\hline & Walking & $\begin{array}{l}\text { MD }(95 \% \text { CI }) \\
\text { Effect Size }\end{array}$ & $\begin{array}{c}-17.65(-37.34,2.05) \\
-0.33\end{array}$ & $\begin{array}{c}-20.66(-39.82,-1.50) \\
-0.39\end{array}$ & $\begin{array}{c}-12.53(-27.43,2.37) \\
-0.30\end{array}$ & $\begin{array}{c}-15.34(-30.01,-0.68) \\
-0.38\end{array}$ & $487.13(39.04)$ \\
\hline & Jogging & $\begin{array}{l}\text { MD }(95 \% \text { CI }) \\
\text { Effect Size }\end{array}$ & $\begin{array}{c}78.44(37.47,119.41) \\
0.69\end{array}$ & $\begin{array}{c}50.84(28.97,72.71) \\
0.84\end{array}$ & $\begin{array}{c}60.09(36.91,83.28) \\
0.93\end{array}$ & $\begin{array}{c}56.78(32.49,81.07) \\
0.84\end{array}$ & $700.37(62.5)$ \\
\hline & Sports & $\begin{array}{c}\text { MD }(95 \% \text { CI }) \\
\text { Effect Size }\end{array}$ & $\begin{array}{c}-72.28(-113.37 \\
-31.19) \\
-0.63\end{array}$ & $\begin{array}{c}-93.69(-134.25 \\
-53.13) \\
-0.83\end{array}$ & $-10.19(-41.31,20.93)$ & $-17.38(-49.82,15.07)$ & $659.03(95.09)$ \\
\hline
\end{tabular}

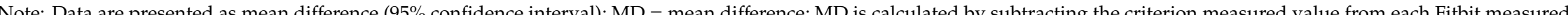
value; $\mathrm{LD}=$ left distal; $\mathrm{LP}=$ left proximal; $\mathrm{RD}=$ right distal; $\mathrm{RP}=$ right proximal; Sample sizes $=31-32$; The bold numbers indicate significant difference from zero $(p<0.05)$. 
Table 6. Comparisons of Calories Estimated by Four Fitbits with Criterion-Measured Calories in the Laboratory and Free-Living Settings.

\begin{tabular}{|c|c|c|c|c|c|c|c|}
\hline & \multirow{2}{*}{ Settings \& Activities } & \multirow{2}{*}{ Variables } & \multicolumn{2}{|c|}{ Right Wrist } & \multicolumn{2}{|c|}{ Left Wrist } & \multirow[b]{2}{*}{ Criterion Measure } \\
\hline & & & Proximal (RP) & Distal (RD) & Proximal (LP) & Distal (LD) & \\
\hline \multirow{5}{*}{ Lab Setting } & Resting & $\begin{array}{l}\text { MD }(95 \% \text { CI }) \\
\text { Effect Size }\end{array}$ & $0.34(-0.88,1.57)$ & $10.44(-11.52,32.39)$ & $\begin{array}{c}-0.28(-0.88,0.31) \\
-0.17\end{array}$ & $\begin{array}{c}-0.03(-0.71,0.65) \\
-0.02\end{array}$ & $4.59(0.95)$ \\
\hline & Slow walking ( $2 \mathrm{mph})$ & $\begin{array}{l}\text { MD }(95 \% \text { CI }) \\
\text { Effect Size }\end{array}$ & $\begin{array}{c}8.48(6.48,10.49) \\
1.55\end{array}$ & $\begin{array}{c}10.00(7.77,12.23) \\
1.62\end{array}$ & $\begin{array}{c}9.47(7.07,11.87) \\
1.42\end{array}$ & $\begin{array}{c}9.88(7.27,12.48) \\
1.37\end{array}$ & $12.09(2.84)$ \\
\hline & Brisk walking(4 mph) & $\begin{array}{l}\text { MD }(95 \% \text { CI }) \\
\text { Effect Size }\end{array}$ & $\begin{array}{c}7.09(4.52,9.66) \\
0.99\end{array}$ & $\begin{array}{c}8.06(4.67,11.46) \\
0.86\end{array}$ & $\begin{array}{c}7.91(4.34,11.47) \\
0.80\end{array}$ & $\begin{array}{c}9.66(5.26,14.05) \\
0.79\end{array}$ & $21.56(4.78)$ \\
\hline & Jogging(6 mph) & $\begin{array}{l}\text { MD }(95 \% \text { CI }) \\
\text { Effect Size }\end{array}$ & $\begin{array}{c}5.28(0.75,9.81) \\
0.42\end{array}$ & $\begin{array}{c}6.77(0.17,13.38) \\
0.38\end{array}$ & $\begin{array}{c}18.69(-7.83,45.2) \\
0.25\end{array}$ & $\begin{array}{c}7.77(1.44,14.11) \\
0.45\end{array}$ & $34.72(7.87)$ \\
\hline & Recovery & $\begin{array}{l}\text { MD }(95 \% \text { CI }) \\
\text { Effect Size }\end{array}$ & $\begin{array}{c}9.38(5.10,13.65) \\
0.79\end{array}$ & $\begin{array}{c}18.81(-1.38,39.01) \\
0.34\end{array}$ & $\begin{array}{c}10.81(3.45,18.17) \\
0.54\end{array}$ & $\begin{array}{c}7.56(3.24,11.88) \\
0.63\end{array}$ & $17.34(3.83)$ \\
\hline \multirow{10}{*}{ Free-living Setting } & Lying down & $\begin{array}{l}\text { MD (95\% CI) } \\
\text { Effect Size }\end{array}$ & $\begin{array}{c}14.28(11.41,17.14) \\
1.80\end{array}$ & $\begin{array}{c}15.03(11.92,18.13) \\
1.77\end{array}$ & $\begin{array}{c}15.00(11.04,18.95) \\
1.39\end{array}$ & $\begin{array}{c}14.83(11.03,18.64) \\
1.43\end{array}$ & $1.35(2.11)$ \\
\hline & Shopping & $\begin{array}{l}\text { MD }(95 \% \text { CI }) \\
\text { Effect Size }\end{array}$ & $\begin{array}{c}7.65(4.56,10.74) \\
0.93\end{array}$ & $\begin{array}{c}8.60(5.98,11.22) \\
1.18\end{array}$ & $\begin{array}{c}10.35(1.90,18.81) \\
0.45\end{array}$ & $\begin{array}{c}7.63(4.26,11.01) \\
0.83\end{array}$ & $6.09(5.62)$ \\
\hline & Climbing stairs & $\begin{array}{l}\text { MD }(95 \% \text { CI }) \\
\text { Effect Size }\end{array}$ & $\begin{array}{c}6.86(3.25,10.47) \\
0.69\end{array}$ & $\begin{array}{c}16.05(1.60,30.49) \\
0.40\end{array}$ & $\begin{array}{c}6.89(3.95,9.83) \\
0.85\end{array}$ & $\begin{array}{c}23.80(-0.28,47.87) \\
0.36\end{array}$ & $29.42(10.97)$ \\
\hline & Laundry & $\begin{array}{l}\text { MD }(95 \% \text { CI }) \\
\text { Effect Size }\end{array}$ & $\begin{array}{c}14.32(9.46,19.19) \\
1.06\end{array}$ & $\begin{array}{c}15.78(10.53,21.04) \\
1.10\end{array}$ & $\begin{array}{c}13.30(7.79,18.81) \\
0.88\end{array}$ & $\begin{array}{c}14.80(8.96,20.64) \\
0.93\end{array}$ & $19.21(10.05)$ \\
\hline & Cleaning & $\begin{array}{l}\text { MD }(95 \% \text { CI }) \\
\text { Effect Size }\end{array}$ & $\begin{array}{c}7.89(2.97,12.81) \\
0.60\end{array}$ & $\begin{array}{c}10.59(5.53,15.66) \\
0.75\end{array}$ & $\begin{array}{c}9.94(4.64,15.23) \\
0.68\end{array}$ & $\begin{array}{c}11.44(5.77,17.10) \\
0.73\end{array}$ & $14.78(12.13)$ \\
\hline & Computer & $\begin{array}{l}\text { MD }(95 \% \text { CI }) \\
\text { Effect Size }\end{array}$ & $\begin{array}{c}\mathbf{1 5 . 3 6}(\mathbf{1 2 . 1 8}, \mathbf{1 8 . 5 4 )} \\
1.74\end{array}$ & $\begin{array}{c}\mathbf{1 6 . 5 2}(\mathbf{1 2 . 3 6 , 2 0 . 6 7 )} \\
1.43\end{array}$ & $\begin{array}{c}\mathbf{1 6 . 6 7}(\mathbf{1 2 . 4 0 , 2 0 . 9 5 )} \\
1.41\end{array}$ & $\begin{array}{c}\mathbf{1 7 . 5 8}(\mathbf{1 2 . 4 2 ,} \mathbf{2 2 . 7 4 )} \\
1.25\end{array}$ & $1.86(1.99)$ \\
\hline & Stretching & $\begin{array}{l}\text { MD }(95 \% \text { CI }) \\
\text { Effect Size }\end{array}$ & $\begin{array}{c}4.28(1.42,7.15) \\
0.55\end{array}$ & $\begin{array}{c}5.72(2.12,9.32) \\
0.57\end{array}$ & $\begin{array}{c}5.35(2.93,7.76) \\
0.80\end{array}$ & $\begin{array}{c}6.04(2.51,9.56) \\
0.62\end{array}$ & $11.9(4.57)$ \\
\hline & Walking & $\begin{array}{l}\text { MD }(95 \% \text { CI }) \\
\text { Effect Size }\end{array}$ & $\begin{array}{c}\mathbf{1 4 . 2 6}(\mathbf{1 1 . 1 6}, \mathbf{1 7 . 3 7 )} \\
1.65\end{array}$ & 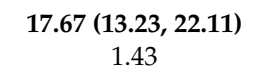 & $\begin{array}{c}\mathbf{1 6 . 6 7}(\mathbf{1 2 . 7 2 , 2 0 . 6 2 )} \\
1.52\end{array}$ & $\begin{array}{c}\mathbf{1 8 . 2 3}(\mathbf{1 3 . 6 3}, \mathbf{2 2 . 8 4 )} \\
1.43\end{array}$ & $19.11(13.08)$ \\
\hline & Jogging & $\begin{array}{l}\text { MD }(95 \% \text { CI }) \\
\text { Effect Size }\end{array}$ & $\begin{array}{c}15.45(-29.41,60.31) \\
0.12\end{array}$ & $\begin{array}{c}-7.36(-14.09,-0.63) \\
-0.39\end{array}$ & $\begin{array}{c}3.80(-9.68,17.27) \\
0.10\end{array}$ & $\begin{array}{c}0.05(-7.86,7.95) \\
0.00\end{array}$ & $59.24(14.45)$ \\
\hline & Sports & $\begin{array}{l}\text { MD }(95 \% \text { CI }) \\
\text { Effect Size }\end{array}$ & $\begin{array}{c}7.08(2.13,12.03) \\
0.52\end{array}$ & $\begin{array}{c}11.91(5.73, \mathbf{1 8 . 0 9 )} \\
0.71\end{array}$ & $\begin{array}{c}11.22(5.27,17.17) \\
0.69\end{array}$ & $\begin{array}{c}13.90(6.56,21.24) \\
0.68\end{array}$ & $48.01(13.25)$ \\
\hline
\end{tabular}

Note: Data are presented as mean difference ( $95 \%$ confidence interval); MD = mean difference; MD is calculated by subtracting criterion measured value from each Fitbit measured value; $\mathrm{LD}=$ left distal; $\mathrm{LP}=$ left proximal; $\mathrm{RD}=$ right distal; $\mathrm{RP}=$ right proximal; Sample sizes $=31-32$; The bold numbers indicate significant difference from zero $(p<0.05)$. 


\section{Discussion}

The major findings of this study were that Fitbit HR placement does not significantly influence the measurements of heart rates and calories in the laboratory and free-living settings. However, there was a significant difference in the step counts measured between the left and right locations while climbing stairs and participating in sports activities. The accuracy of heart rate and step-count estimates were moderate in some light-intensity activities, but it was poor during moderate- to vigorous-intensity activities. Calorie estimates were poor, and they were overestimated both in the laboratory and free-living conditions.

We found that heart rate and calorie estimations were not different, while some differences were observed in step counts. Step counts were reported as higher at the left wrist (LP, LD) than the right wrist (RP, RD) during claiming stairs and sports activities. A previous study examined the inter-device reliability of Fitbit HR estimated step counts between dominant and non-dominant wrist during normal walking [11]. They found no significant differences in step counts between the locations, and the inter-device reliability between dominant and non-dominant wrist was good (inter-correlation coefficient $=0.81$, confidence interval, 0.63 to 0.90 ). It is unclear why significant differences in step counts were detected during climbing stairs and sports activities in the present study. It was assumed that different axis motion between right and left arms with vigorous activity might affect locations' disparities. For instance, participants performed basketball dribbling as a sports activity in this study. While the dribbling hand moved with a sagittal motion, the other hand remained free of motion. This unilateral hand movement may cause the difference in step counts between the right and left hands.

A number of studies have been conducted to examine the accuracy of heart rate sensors during exercise or free-living activities [5,6,22]. Stahl et al. reported that wrist-worn activity monitors (i.e., Mio Alpha, TomTom, Microsoft Band, Basis Peak, and Fitbit HR) were strongly correlated with a criterion measure ranged from 0.87 to 0.96 in resting, walking, and running (i.e., 3.2, 4.8, 6.4, 8.0, $9.6 \mathrm{~km} / \mathrm{h}$ ) [5]. The authors pointed out that a wrist-worn activity monitor could estimate heart rate more accurately when participants swung their arm during walking and jogging. Wallen et al. also reported that there was a strong correlation in heart rate estimation between Fitbit HR and reference methods (i.e., electrocardiograph) during walking, running, and cycling (mean difference: -9.3 to $8.5 \mathrm{bpm}, \mathrm{r}=0.81$ ) [22].

However, our findings differed from the previous studies where the accuracy decreased during moderate- to vigorous-intensity activities (Table 4). Gorny et al. support our results that Fitbit HR underestimated the heart rate during light intensity activities $(-4.22 \mathrm{bpm})$, but this error increased during the moderate to vigorous activities $(-16.2 \mathrm{bpm})$ in a free-living setting [6]. This conflict in results between studies may be influenced by various factors such as emotional status, skin color, and locations (i.e., dominant vs. non-dominant, proximal vs. distal, wrist circumference). Although different Fitbit HR placements did not affect heart rate estimation in the present study (Table 3), interesting trends were observed in the present study where the Fitbit HR worn at the proximal location showed fewer errors than when worn at the distal position during moderate- to vigorous-intensity activities. This trend partially supports the company's instruction where Fitbit HR captures heart rate estimation more accurately at the higher location (i.e., up to three-finger width) during exercise [23]. However, significant error compared with criterion measure failed to reflect heart rate estimation accuracy during moderate- to vigorous-intensity activities.

The accuracy of Fitbit HR step counts was moderate during brisk walking and jogging in the laboratory setting, but the accuracy decreased during slow walking on the treadmill and most of the free-living activities. A systematic review study demonstrated that the overall accuracy of Fitbit monitors was high in step counts (mean correlation $\geq 0.80$ ), while the accuracy decreased during slow walking, which supports our results [12]. Relatively poor accuracy was identified across all Fitbit HR locations during shopping, laundry, and jogging activities in a free-living setting. 
In the present study, the accuracy of calorie estimates was poor. Fitbit HR overestimated calories regardless of its position and activity types. Physical activity is commonly defined as "any bodily movement produced by skeletal muscles that require energy expenditure." [24]. Although the understanding of energy expenditure during free-living activities is important, many monitors failed to provide accurate calorie estimations [10]. Brooke et al. reported that Fitbit HR overestimated calories ( $334.9 \mathrm{kcal}$ ) under free-living conditions, but the monitor provided reasonable calorie estimates compared to other activity monitors (i.e., Polar Loop, Misfit Shine, and Garmin VivoFit) [9]. There is an important consideration when comparing the data with previous studies related to different criterion measures used in the studies. For instance, while our study used ActiGraph GT3X, other studies used SenseWear Armband Mini, ActiheartTM and BodyMedia Core as criterion measures $[9,10]$. Although many research-grade devices have shown high correlations with indirect calorimetry, it is still debatable whether those can be accepted as criterion measures.

The major strength of the current study is the research design, which enables us to establish evidence of both inter-device reliability and validity of Fitbit HR's PA measurements. The study collects all three common PA measurements: heart rate, calories, and step counts, which is also a strength of the study. Although carefully designed, this study is not free of limitations. The study sample was comprised of healthy young adults. Different activity levels of the population may influence the difference in measurements among wearing placement. Results may not be generalizable to other populations, particularly the clinical population. Another potential limitation is that the sample is dominantly right-handed, so we could not compare left-handed and right-handed participants. More research is needed to consider how handedness affects the study outcomes. Secondly, this study may limit generalizing the overall results of fitness trackers. It is well known that different algorisms built into each fitness tracker can create different outcomes [5]. However, we believe that our results may provide substantial information to researchers or consumers when considering the use of Fitbit devices such as Fitbit HR in the research or clinical environments. This is because placement has become an important consideration to accurately monitor people's PA levels as the number of wrist-worn fitness trackers users increases. Especially, the PPG sensor can create a great variation of heart rate outcomes based on the place (i.e., proximal vs. distal; dominant vs. non-dominant) applied even though the same device is used [25]. Nevertheless, future studies testing various types of fitness trackers are required to generalize the PA outcomes.

Lastly, we chose ten different activities as representative of the free-living condition in this study. These activities may not fully represent the type of physical activity by young adults. However, we have a broad range of activities ranging from sedentary to vigorous activities with a balanced number of activities.

\section{Conclusions}

This study revealed that Fitbit HR placement does not have a significant influence on the measurements of heart rate and calories during the laboratory and free-living settings. However, there was a significant difference in the step counts measured between the left and right locations while climbing stairs and during sports activities. The accuracy of heart rate and step-count estimations were moderate in some light intensity activities, but the error grew during moderate- to vigorous-intensity activities. Fitbit HR overestimated the calories regardless of their positions and activity types. The results demonstrated that different placement of Fitbit HR does not influence the measurement outcomes, but the device failed to satisfy accuracy against the criterion measure and this error increased for moderate- to vigorous-intensity activities. Given the nature of our study with a limited fitness tracker application, future research is warranted to generalize the placement effect of wrist-worn activity monitors on PA outcomes such as heart rate, step count, and calories. 
Author Contributions: Conceptualization, H.C.J., N.H.L., and S.L.; investigation, N.H.L., S.J.; methodology, H.C.J., N.H.L., M.K., S.L.; data curation. M.K.; project administration, S.L.; supervision, S.L.; writing-original draft preparation, H.C.J.; writing-review and editing, H.C.J., M.K., S.L. All authors have read and agreed to the published version of the manuscript.

Funding: This research received no external funding.

Acknowledgments: The authors would like to thank all participants for their valuable time and for their voluntary participation.

Conflicts of Interest: No conflict of interest existed in this study.

\section{References}

1. Blair, S.N. Physical inactivity: The biggest public health problem of the 21st century. Br. J. Sports Med. 2009, 43, 1-2. [PubMed]

2. Sofi, F.; Capalbo, A.; Cesari, F.; Abbate, R.; Gensini, G.F. Physical activity during leisure time and primary prevention of coronary heart disease: An updated meta-analysis of cohort studies. Eur. J. Cardiov. Prev. R. 2008, 15, 247-257. [CrossRef] [PubMed]

3. CCS Insight. Wearable Market to be Worth $\$ 25$ Billion by 2019. Available online: https://www.ccsinsight.com/ press/company-news/2332-wearables-market-to-be-worth-25-billion-by-2019-reveals-ccs-insight/ (accessed on 20 January 2019).

4. Statista. Most Popular Health and Fitness Apps in The United State as of May 2018 by Monthly Active Users. Available online: https://www.statista.com/statistics/650748/health-fitness-app-usage-usa/ (accessed on 20 January 2020).

5. Stahl, S.E.; An, H.S.; Dinkel, D.M.; Noble, J.M.; Lee, J.M. How accurate are the wrist-based heart rate monitors during walking and running activities? Are they accurate enough? BMJ Open Sport Exerc. Med. 2016, 2, 1-7. [CrossRef] [PubMed]

6. Gorny, A.W.; Liew, S.J.; Tan, C.S.; Müller-Riemenschneider, F. Fitbit Charge HR Wireless Heart Rate Monitor: Validation study conducted under free-living conditions. JMIR mHealth uHealth 2017, 5, 157. [CrossRef] [PubMed]

7. Maher, C.A.; Lewis, L.K.; Ferrar, K.; Marshall, S.; De Bourdeaudhuij, I.; Vandelanotte, C. Are health behavior change interventions that use online social networks effective? A systematic review. JMIR 2014, 16, 40. [CrossRef] [PubMed]

8. Sullivan, A.N.; Lachman, M.E. Behavior change with fitness technology in sedentary adults: A review of the evidence for increasing physical activity. Front. Public Health 2017, 4, 289. [CrossRef]

9. Brooke, S.M.; An, H.S.; Kang, S.K.; Noble, J.M.; Berg, K.E.; Lee, J.M. Concurrent validity of wearable activity trackers under free-living conditions. J. Strength Cond. Res. 2017, 31, 1097-1106. [CrossRef]

10. Chowdhury, E.A.; Western, M.J.; Nightingale, T.E.; Peacock, O.J.; Thompson, D. Assessment of laboratory and daily energy expenditure estimates from consumer multi-sensor physical activity monitors. PLoS ONE 2017, 12, 0171720. [CrossRef]

11. De Man, M.; Vanderploeg, E.; Aimers, N.; MacMahon, C.; Wise, L.; Parrington, L. Validity and inter-device reliability of dominant and non-dominant wrist worn activity trackers in suburban walking. Sensoria $A J$. Mind Brain Cult. 2016, 12, 40-46. [CrossRef]

12. Evenson, K.R.; Goto, M.M.; Furberg, R.D. Systematic review of the validity and reliability of consumer-wearable activity trackers. IJBNPA 2015, 12, 159. [CrossRef]

13. Chen, M.D.; Kuo, C.C.; Pellegrini, C.A.; Hsu, M.J. Accuracy of Wristband Activity Monitors during Ambulation and Activities. Med. Sci. Sports Exer. 2016, 48, 1942-1949. [CrossRef] [PubMed]

14. Chu, A.H.; Ng, S.H.; Paknezhad, M.; Gauterin, A.; Koh, D.; Brown, M.S.; Müller-Riemenschneider, F. Comparison of wrist-worn Fitbit Flex and waist-worn ActiGraph for measuring steps in free-living adults. PLoS ONE 2017, 12, 0172535. [CrossRef] [PubMed]

15. Nelson, M.B.; Kaminsky, L.A.; Dickin, D.C.; Montoye, A.H. Validity of consumer-based physical activity monitors for specific activity types. Med. Sci. Sports Exerc. 2016, 48, 1619-1628. [CrossRef] [PubMed]

16. Jackson, A.S.; Pollock, M.L. Generalized equations for predicting body density of men. Br. J. Nutr. 1978, 40, 497-504. [CrossRef] [PubMed]

17. Siri, W.E. Body composition from fluid spaces and density: Analysis of methods 1961. Nutrition 1993, $19,480$. 
18. Barreira, T.V.; Kang, M.; Caputo, J.L.; Farley, R.S.; Renfrow, M.S. Validation of the Actiheart monitor for the measurement of physical activity. Int. J. Exerc. Sci. 2009, 2, 7.

19. Ainsworth, B.E.; Haskell, W.L.; Herrmann, S.D.; Meckes, N.; Bassett, J.D.R.; Tudor-Locke, C.; Leon, A.S. 2011 Compendium of Physical Activities: A second update of codes and MET values. Med. Sci. Sports Exerc. 2011, 43, 1575-1581. [CrossRef]

20. Montoye, A.H.; Mudd, L.M.; Biswas, S.; Pfeiffer, K.A. Energy Expenditure Prediction Using Raw Accelerometer Data in Simulated Free Living. Med. Sci. Sports Exerc. 2015, 47, 1735-1746. [CrossRef]

21. Freedson, P.S.; Melanson, E.; Sirard, J. Calibration of the Computer Science and Applications, Inc. accelerometer. Med. Sci. Sports Exerc. 1998, 30, 777-781. [CrossRef]

22. Wallen, M.P.; Gomersall, S.R.; Keating, S.E.; Wisløff, U.; Coombes, J.S. Accuracy of heart rate watches: Implications for weight management. PLoS ONE 2016, 11, 0154420. [CrossRef]

23. Fitbit. Best Practices for Purepulse ${ }^{\mathrm{TM}}$ Accuracy. Available online: https://www.fitbit.com/purepulse-tips (accessed on 29 July 2018).

24. World Health Organization. Physical Activity. Available online: https://www.who.int/health-topics/physicalactivity\#tab=tab_1 (accessed on 5 July 2020).

25. Tamura, T.; Maeda, Y.; Sekine, M.; Yoshida, M. Wearable photoplethysmographic sensors-past and present. Electronics 2014, 3, 282-302. [CrossRef]

(C) 2020 by the authors. Licensee MDPI, Basel, Switzerland. This article is an open access article distributed under the terms and conditions of the Creative Commons Attribution (CC BY) license (http://creativecommons.org/licenses/by/4.0/). 